\title{
Praxis von Internetwahlen
}

Auf den ersten Blick mag das Wahlcomputerurteil von 2009 ein Rückschlag für das Thema,Elektronische Wahlen' in Deutschland gewesen sein. Auf den zweiten Blick hat es aber eine Reihe von Forschungsfragen aufgeworfen, sowohl für parlamentarische als auch für andere Wahlen, wie zum Beispiel Sozial- oder Hochschulwahlen. Aus rechtswissenschaftlicher Sicht stellen sich die Fragen: Welcher Grad an Öffentlichkeit ist bei welcher Wahl erforderlich? Was bedeutet die Entscheidung des Bundesverfassungsgerichts für den gesetzlichen Gestaltungsspielraum, $d$. h. den geforderten Grad der Umsetzung aller Wahlrechtsgrundsätze?

Auf dieser Basis stellt sich aus Sicht der Informatik die Frage, welche Wahltechnik für welche Wahl die Wahlrechtsgrundsätze zu einem ausreichenden Grad erfüllt. Dabei hat sich gezeigt, dass diese Frage nicht ausschließlich auf dem zu Grunde liegenden kryptografischen Protokoll zu beantworten ist, sondern eine einsatzfertige Wahltechnik vorliegen muss. Dies macht aus Sicht der Informatik eine abschließende Analyse schwierig: Einerseits existieren zwar viele theoretische Konzepte, aber kaum einsatzfertige Wahltechniken. Anderseits existiert auf Grund des Gestaltungsspielraums nicht die eine Wahltechnik, sondern eine Vielzahl an zulässigen Wahltechniken, und jede dieser Wahltechniken hat ihre Vor- und Nachteile hinsichtlich des einen oder anderen Wahlrechtsgrundsatzes.

Zusätzlich zu der Auseinandersetzung mit dem gesetzlichen Gestaltungsspielraum (insbesondere dem Öffentlichkeitsgrundsatz) aus rechtswissenschaftlicher Sicht, und zusätzlich zu der Analyse existierender Ansätze und einsatzfertiger Wahltechniken hinsichtlich des gesetzlichen Gestaltungsspielraums stehen elektronischen Wahlen zwei weiteren Herausforderungen gegenüber: Aus rechtswissenschaftlicher Sicht müssen Vorschläge für die Gesetzgebung und aus Sicht der Informatik entsprechende Prüfkonzepte erarbeitet werden.

In dem von der DFG geförderten interdisziplinären Forschungsprojekt ModIWa II haben Wissenschaftler der Universitäten Kassel und Koblenz und der Technischen Universität Darmstadt an diesen Herausforderungen im Kontext von Internetwahlen geforscht. Einen Einblick in die Ergebnisse bietet dieses Schwerpunktheft. Dabei wurden zwei konkrete Wahlen betrachtet: Sozialwahlen und Wahlen an Universitäten, wobei der erste Fall ausführlicher behandelt wird. Beginnend mit einer allgemeinen Analyse von Internetwahlen bei Sozialwahlen wird beispielhaft das Wahlsystem Polyas nach formalen Kriterien hinsichtlich seiner diesbezüglichen Eignung untersucht. In einem dritten Beitrag werden Vorschläge für die Gesetzgebung am Beispiel von Internetwahlen bei Sozialwahlen erarbeitet. Schließlich werden für Hochschulwahlen aus Sicht der Rechtswissenschaft rechtliche Anforderungen an die technische und rechtliche Gestaltung von Internetwahlen bei Hochschulwahlen entwickelt. Der Schwerpunkt schließt mit der Betrachtung möglicher Prüfkonzepte und untersucht dabei insbesondere die Tauglichkeit von Common Criteria-Schutzprofilen für Internetwahlen im Hinblick auf die Flexibilität bei der Definition von Anforderungen, die durch den gesetzlichen Gestaltungspielraum benötigt wird.

Insgesamt zeigt dieses Schwerpunktheft, dass das interdisziplinäre Verständnis und die Anforderungen an Internetwahlen durch das Projekt einen deutlichen Schub bekommen haben. Es zeigt aber auch, dass keine der existierenden Wahltechniken für Sozialwahlen oder gar politische Wahlen geeignet sind, so dass hier auf technischer Seite weiterhin Forschungsbedarf besteht - hinsichtlich der Entwicklung geeigneter Wahltechniken sowie geeigneter Prüfkonzepte.

Katharina Bräunlich, Rüdiger Grimm und Melanie Volkamer 